\title{
Risk factors for development of cervical spondylotic myelopathy: results of a systematic review
}

Authors Anoushka Singh ${ }^{1}$, Lindsay Tetreault ${ }^{2}$, Michael G Fehlings ${ }^{1}$, Dena J Fischer ${ }^{3}$, Andrea C Skelly ${ }^{3}$

Institutions ${ }^{1}$ Division of Neurosurgery and Spinal Program, Toronto Western Hospital, Toronto, Ontario, Canada

${ }^{2}$ Institute of Medical Sciences, University of Toronto, Ontario, Canada

${ }^{3}$ Spectrum Research Inc, Tacoma, WA, USA

\section{ABSTRACT}

Study design: Systematic review.

Study rationale: Cervical spondylotic myelopathy (CSM) is a common cause of spinal cord dysfunction that may be asymptomatic or may present with severe symptoms. Since CSM has an insidious manifestation, identification of risk factors associated with this condition may aid clinicians in monitoring high-risk patients and implementing appropriate management strategies.

Objective: To assess sociodemographic, clinical, radiographic, and genetic risk factors associated with presence of CSM in patients 18 years or older.

Methods: A systematic review of the literature was performed using PubMed, the National Guideline Clearinghouse Databases, and bibliographies of key articles to assess risk factors associated with CSM. Articles were reviewed by two independent reviewers based on predetermined inclusion and exclusion criteria. Each article was evaluated using a predefined quality-rating scheme.

Results: From 486 citations, eight articles met all inclusion and exclusion criteria. Larger vertebral body and smaller spinal canal and Torg/Pavlov ratio were associated with CSM diagnosis, while gender was not associated with a CSM diagnosis across multiple studies. There were inconsistent reports with respect to increased age as a risk factor for CSM diagnosis.

Conclusion: The limited data available suggests that inherent anatomical features that may contribute to congenital cervical stenosis may be associated with CSM. This systematic review is limited by the small number of high-quality studies evaluating prognostic factors for CSM. The overall strength of evidence for all risk factors evaluated is low. 


\section{STUDY RATIONALE AND CONTEXT}

Cervical spondylotic myelopathy (CSM) is the most common cause of spinal cord dysfunction in patients 55 years or older. This disease is caused by the degeneration of various components of the vertebra including the vertebral body, intervertebral disc, supporting ligaments, and the facet and other true joints. These anatomical changes, specifically the development of osteophytic spurs, may lead to the narrowing of the spinal canal and potentially to mechanical compression of the neural elements. Long-standing compression of the spinal cord, in turn, can result in irreversible damage including demyelination and necrosis of the gray matter. The onset of CSM is insidious and usually progresses in a stepwise fashion. Furthermore, CSM may be asymptomatic or may present with a wide range of symptoms, from numb clumsy hands to severe gait impairment $[1,2]$. Since CSM has an insidious manifestation, it is essential to determine risk factors associated with this condition. Identification of these factors will allow clinicians to monitor their high-risk patients and implement appropriate management strategies.

\section{OBJECTIVE}

To assess sociodemographic, patient, behavioral, environmental, or inborn risk factors associated with the presence of CSM in patients 18 years or older.

Fig 1 Results of literature search.

1. Total citations from search $(n=486)$

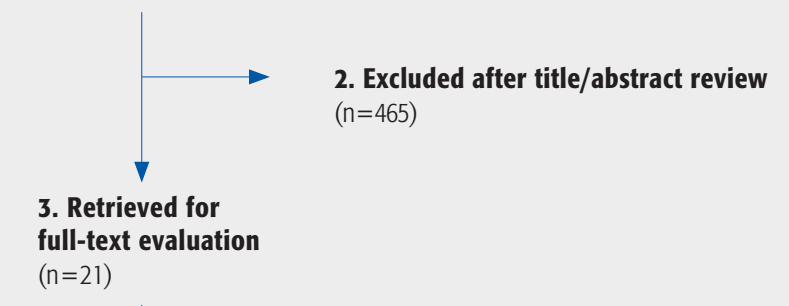

$(n=21)$

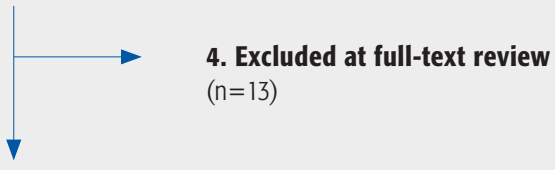

5. Publications included $(n=8)$

\section{MATERIALS AND METHODS}

Study design: Systematic review.

Search: PubMed and National Guideline Clearinghouse Databases; bibliographies of key articles (Fig 1).

Dates searched: 1950 through December 2011.

Inclusion criteria: Patients diagnosed with CSM. Studies explicitly designed to evaluate risk factors (sociodemographic, behaviors, occupational or lifestyle, environmental, inborn or inherited characteristics) for CSM in patients older than 18 years were sought. Studies were considered if CSM and evaluation of risk factors were described in the title and/or abstract. Studies which explicitly compared groups which had CSM with those who did not were considered for inclusion. Only studies in which factors logically preceded (or were measured prior to) development of CSM were included.

Exclusion criteria: Cervical radiculopathy diagnosis, cervical spondylosis only with no myelopathy, thoracic and/or lumbar myelopathy, CSM patients with history of acute trauma or tumor, patients younger than 18 years, factors related to recovery after treatment or progress after treatment; factors that related to criteria for CSM diagnosis, clinical assessment, physiological testing; factors that are along the continuum of spondylosis, degenerative spinal disease/processes or its progression; cost-of-care analyses, case series or case reports.

Risk factors: Sociodemographic, patient characteristics, occupational, lifestyle, behavioral, environmental, congenital, inherited and/or genetic factors for CSM.

Outcomes: Cervical spondylotic myelopathy.

Analysis: Descriptive statistics; statistics and effect estimates as reported by authors.

Details about methods can be found in the Web Appendix at www.aospine.orglebsj 


\section{RESULTS}

The initial search yielded 486 citations, 21 of which underwent full-text review. Eight studies met the inclusion criteria for assessing prognostic factors associated with CSM diagnosis. One study was a poor quality cohort (Level of Evidence [LoE] III) [3], and seven were considered casecontrol studies (LoE III) [4-10]. Additional details regarding the critical appraisal and study exclusion criteria are available in the Web Appendix.

Table 1 describes the characteristics of included studies with criteria used for determining the presence (diagnosis) of CSM. Table 2 summarizes the primary factors evaluated in the studies and effect size estimates reported in the studies.

Table 3 sums up findings for factors assessed across multiple studies. Table 4 reviews factors that were evaluated in only one study.

Prognostic factors (Tables 3 and $\mathbf{4})$

\section{Sociodemographic, patient, and occupational factors}

Only age and gender were evaluated across multiple studies.

- Age: Increased age as a risk factor for CSM was assessed in three studies, two of which found an association between age and diagnosis of CSM.

- In one case-control study older patients were more likely to have CSM compared with subjects with neck pain but no clinical or radiological evidence of CSM based on multivariate analysis $(P=.002)$ [10].

- In one retrospective cohort study increased age was an independent risk factors for CSM in a sub-analysis comparing CSM patients with those without CSM (odds ratio $=1.1$ per year of age; $95 \%$ confidence interval: 1.01-1.14) [3].

- One study [4] had no statistical relationship between age and CSM diagnosis.

- Gender: Female gender was not associated with the presence of CSM across multiple studies [3, 10].

Findings from single studies:

- Number of working years and working in an extensionstrain occupation were not associated with CSM [3].
Inherent or congenital characteristics: characteristics of the spine or spinal canal (based on radiological measurements)

The following measurements were assessed in multiple studies:

- Results across two case-control studies were inconsistent with regard to an association between spinal canal cross-sectional area (CSA) and the presence of CSM [5, 6]. In one study spinal canal CSA was not associated with CSM in a multivariate logistic regression model [5], while in another study smaller spinal canal CSA was associated with CSM in an independent analysis that accounted for sociodemographic and patient factors [6].

- In two case-control studies a larger sagittal diameter of the vertebral body and smaller sagittal diameter of the spinal canal were associated with the presence of CSM $[4,6]$. In another study these measurements were associated with CSM in independent analyses that accounted for sociodemographic and patient factors [6].

- In two case-control studies a smaller transverse diameter of the spinal canal was associated with CSM [6, 7]. In one study this spinal canal measurement was associated with CSM in an analysis that accounted for sociodemographic and patient factors [6].

- In two studies a smaller Torg/Pavlov ratio was associated with the presence of CSM [4, 10]. In a case-control study, smaller mean Torg/Pavlov ratios were linked with CSM in a multivariate logistic regression model $(P<.0001)[10]$.

Findings from isolated studies included:

- Smaller CSA of cerebrospinal fluid space [5]; larger vertebral body transverse diameter and CSA, larger sagittal and transverse vertebral body/spinal canal ratios, smaller sagittal and transverse space available for the spinal cord (SAC) [6]; and higher canal-occupying ratio of the spinal cord [7] were associated with CSM in single studies.

- Cross-sectional SAC [6] and dural tube transverse area [7] were not related with CSM in single studies.

\section{Inherited (genetic) factors}

- Inherited factors were not evaluated across multiple studies.

- In isolated studies, the following associations with CSM were reported:

- Having relatives with CSM [8] and vitamin D receptor gene polymorphism [9] were linked with the presence of CSM in single studies. 
Table 1 Characteristics of studies reporting prognostic factors for cervical spondylotic myelopathy (CSM).*

\begin{tabular}{|c|c|c|c|c|c|c|}
\hline Author & Study design & Demographics & Disease/case definition & $\begin{array}{l}\text { Study population } \\
\text { characteristics }\end{array}$ & $\mathbf{F} / \mathbf{U}, \%$ & CoE \\
\hline \multicolumn{7}{|c|}{ Poor quality studies (CoE III), controlled for extraneous prognostic factors } \\
\hline $\begin{array}{l}\text { Golash et al } \\
{[5](2001)^{\dagger}}\end{array}$ & Case control & $\begin{array}{l}\mathrm{N}=30 \\
\text { Female: } 43 \% \\
\text { Mean age, } \mathrm{y}: \\
39 \pm 2.2(\mathrm{Gr} 1) \\
39 \pm 1.5(\operatorname{Gr} 2)\end{array}$ & $\begin{array}{l}\text { Symptoms and signs of CSM based on } \\
\text { clinical and x-ray findings; myelopathy } \\
\text { was assessed clinically based on } \\
\text { increased tone, hypereflexia, } \\
\text { decreased power, sensory loss, } \\
\text { extensor plantar response }\end{array}$ & $\begin{array}{l}\text { Group 1: CSM }(\mathrm{N}=20) \\
\text { Group 2: Normal controls without } \\
\text { symptoms or signs of spondylosis } \\
\text { or myelopathy }(\mathrm{N}=10)\end{array}$ & NR & III \\
\hline $\begin{array}{l}\text { Hukuda et } \\
\text { al [6] (1996) }\end{array}$ & Case control & $\begin{array}{l}\mathrm{N}=85 \\
\text { Female: 44\% } \\
\text { Mean age, } \mathrm{y}: \\
56 \text { (range, 22-75; Gr 1) } \\
52 \text { (range, 22-80; } \operatorname{Gr} 2 \text { ) }\end{array}$ & $\begin{array}{l}\text { Diagnosis of CSM through } \\
\text { CT-myelography and satisfying } \\
\text { qualification of classic myelopathy:; } \\
\text { myelopathy was due to cervical } \\
\text { spondylosis or OPLL }\end{array}$ & $\begin{array}{l}\text { Group 1: diagnosis of CSM }(\mathrm{N}=61) \\
\text { Group 2: subjects with spinal } \\
\text { lesions other than } \operatorname{CSM}^{\S}(\mathrm{N}=24)\end{array}$ & NR & III \\
\hline $\begin{array}{l}\text { Patel et al } \\
\text { [8] (2012) }\end{array}$ & Case control & $\begin{array}{l}\mathrm{N}=1,486 \\
\text { Female: NR } \\
\text { Age: NR }\end{array}$ & $\begin{array}{l}\text { Diagnosis of CSM through registry } \\
\text { (ICD-9: 721.1) }\end{array}$ & $\begin{array}{l}\text { Group 1: diagnosis of CSM } \\
\text { (N=486) } \\
\text { Group 2: gender-, age- and } \\
\text { birthplace-matched controls } \\
(\mathrm{N}=1000)\end{array}$ & NR: $100 \%$ & III \\
\hline $\begin{array}{l}\text { Takamiya et } \\
\text { al [3] (2006) }\end{array}$ & $\begin{array}{l}\text { Retrospective } \\
\text { cohort }\end{array}$ & $\begin{array}{l}\mathrm{N}=368 \\
\text { Female: } 54 \% \\
\text { Mean age, y: } \\
51.0 \text { (range, 30-69; Gr 1) } \\
50.8 \text { (range, 30-69; Gr 2) }\end{array}$ & $\begin{array}{l}\text { Diagnosis of cervical myelopathy } \\
\text { based on clinical presentation of } \\
\text { numbness of bilateral fingers and no } \\
\text { other neurological diseases }\end{array}$ & $\begin{array}{l}\text { Group 1: occupation working in } \\
\text { cervical extension strain position } \\
\geq 8 \mathrm{~h} / \text { day, } 8 \mathrm{mo} / \mathrm{y}(\mathrm{N}=177) \\
\text { Group 2: did not work in cervical } \\
\text { extension strain position }(\mathrm{N}=191)\end{array}$ & NR & III \\
\hline $\begin{array}{l}\text { Wang et [9] } \\
(2010)\end{array}$ & Case control & $\begin{array}{l}\mathrm{N}=297 \\
\text { Female: } 39 \% \\
\text { Mean age, } \mathrm{y}: \\
45.4 \pm 3.5(\mathrm{Gr} 1) \\
46.1 \pm 2.8(\mathrm{Gr} 2)\end{array}$ & $\begin{array}{l}\text { Diagnosis of CSM through } \\
\text { examination (including modified JOA } \\
\text { score) and MRI imaging; excluded } \\
\text { subjects with congenital cervical } \\
\text { anomalies, trauma, OPLL, ankylosing } \\
\text { spondylitis, cervical inflammatory } \\
\text { disease }\end{array}$ & $\begin{array}{l}\text { Group 1: diagnosis of CSM } \\
(\mathrm{N}=144) \\
\text { Group 2: gender- and } \\
\text { aged-matched controls with } \\
\text { negative MRI findings }(N=153)\end{array}$ & NR: $100 \%$ & III \\
\hline $\begin{array}{l}\text { Yue et al } \\
{[10](2001)}\end{array}$ & Case control & $\begin{array}{l}\mathrm{N}=116 \\
\text { Female: } 44 \% \\
\text { Age range, y: } \\
29-77(\mathrm{Gr} 1) \\
16-60(\mathrm{Gr} 2)\end{array}$ & $\begin{array}{l}\text { Diagnosis of CSM through x-ray } \\
\text { findings (CT-myelography or MRI) } \\
\text { and neurological examination; } \\
\text { excluded subjects with myelopathy } \\
\text { secondary to trauma, OPLL }\end{array}$ & $\begin{array}{l}\text { Group 1: diagnosis of CSM }(\mathrm{N}=28) \\
\text { Group 2: controls with negative } \\
\text { neurological examination and } \mathrm{x} \text {-ray } \\
\text { findings }(\mathrm{N}=88)\end{array}$ & NR: $100 \%$ & III \\
\hline \multicolumn{7}{|c|}{ Poor quality studies (CoE III), did not control for extraneous prognostic factors } \\
\hline $\begin{array}{l}\text { Chen et al } \\
\text { [4] (1994) }\end{array}$ & Case control & $\begin{array}{l}\mathrm{N}=200 \\
\text { Female: 0\% } \\
\text { Mean age: NR }\end{array}$ & $\begin{array}{l}\text { Diagnosis of CSM through } \\
\text { neurological symptoms and cervical } \\
\text { myelography, CT or MRI imaging; had } \\
\text { undergone decompressive } \\
\text { procedures for cervical myelopathy; } \\
\text { excluded myelopathy due to trauma, } \\
\text { disc herniation, upper cervical } \\
\text { disorders }\end{array}$ & $\begin{array}{l}\text { Group 1: male subjects, diagnosis } \\
\text { of CSM }(N=100) \\
\text { Group 2: gender- and } \\
\text { aged-matched controls }(N=100)\end{array}$ & NR & III \\
\hline $\begin{array}{l}\text { Okada et al } \\
\text { [7] (1994) }\end{array}$ & Case control & $\begin{array}{l}\mathrm{N}=170 \\
\text { Female: } 42 \% \\
\text { Mean age, } \mathrm{y}: \\
60.5 \text { (range, } 39-84 ; \operatorname{Gr} 1) \\
46.5 \text { (range, } 21-73 ; \text { Gr 2) }\end{array}$ & $\begin{array}{l}\text { Diagnosis of CSM through } \\
\text { neurological examination (JOA score) } \\
\text { and radiographic findings }\end{array}$ & $\begin{array}{l}\text { Group 1: diagnosis of CSM }(n=74) \\
\text { Group 2: healthy controls with neck } \\
\text { pain and negative neurological } \\
\text { examination }(n=96)\end{array}$ & NR & III \\
\hline
\end{tabular}

* F/U indicates follow-up; NR, not reported; with regard to percentage follow-up, NR shows that this was not reported or could not be determined as the number of eligible patients and/or number lost to follow-up or without data could not be determined; Gr, group; CT, computed tomography; ICD, International Classification of Diseases; JOA, Japanese Orthopedics Association; MRI, magnetic resonance imaging; OPLL, Ossification of posterior longitudinal ligament. Characteristics were reported that related to study question.

† Golash et al [5] (2001): Study population also included a group of subjects with symptoms suggestive of cervical spondylosis, although this group did not meet the inclusion criteria for this systematic review.

‡ Hakuda et al [6] (1996): Classic myelopathy defined as transverse or Brown-Sequard type by Crandall and Batzdorf classification.

$\S$ Hakuda et al [6] (1996): Control population included 4 subjects with metastatic thoracic tumors; 3 thoracic cord tumor; 3 rheumatoid spondylitis: 3 traumatic subluxation of the cervical spine; 2 ossification of the ligamentum flavum of the thoracic spine, thoracic disc herniation; 1 anterior spinal artery syndrome; 1 traumatic thoracic spine dislocation; 1 spinal process fracture of the cervical spine; 1 flexion-extension injury of the cervical spine; 1 cervical spondylotic radiculopathy; and 2 unknown. 
Table 2 Prognostic factors for CSM and outcomes evaluated.*

\begin{tabular}{|c|c|c|}
\hline Study & Potential prognostic factors evaluated & Significant results ${ }^{\dagger}$ \\
\hline \multicolumn{3}{|c|}{ Poor quality studies (COE III), controlled for extraneous prognostic factors } \\
\hline $\begin{array}{l}\text { Golash et al [5] } \\
(2001)\end{array}$ & $\begin{array}{l}\text { CSA of spinal canal } \\
\text { CSA of CSF space }\end{array}$ & $\begin{array}{l}\text { Associations with CSM diagnosis (compared with controls): } \\
\text { Smaller CSA of CSF space }(P<.02)\end{array}$ \\
\hline $\begin{array}{l}\text { Hukuda et al [6] } \\
\text { (1996) }\end{array}$ & $\begin{array}{l}\text { Transverse diameter of vertebral body } \\
\text { Sagittal diameter of vertebral body } \\
\text { CSA of vertebral body } \\
\text { Transverse diameter of spinal canal } \\
\text { Sagittal diameter of spinal canal } \\
\text { CSA of spinal canal } \\
\text { Ratio between vertebral body and spinal canal (sagittal } \\
\text { diameter) } \\
\text { Ratio between vertebral body and spinal canal } \\
\text { (transverse diameter) } \\
\text { Sagittal SAC } \\
\text { Transverse SAC } \\
\text { Cross-sectional SAC }\end{array}$ & $\begin{array}{l}\text { Associations with CSM diagnosis (compared with controls): } \\
\text { - Larger vertebral body transverse diameter (except } C 4 ; P<.03 \text { to } P<.0001 \text { ) } \\
\text { - Larger vertebral body sagittal diameter (all levels; } P=.03 \text { to } P<.0001 \text { ) } \\
\text { - Larger vertebral body CSA (except } C 7 ; P<.02 \text { to } P<.0001 \text { ) } \\
\text { - Smaller spinal canal transverse diameter (all levels; } P<.002 \text { to } P<.0001 \text { ) } \\
\text { - Smaller spinal canal sagittal diameter (all levels; } P<.0001 \text { ) } \\
\text { - Smaller spinal canal CSA (C3, } P=.008 ; C 7, P=.001 \text { ) } \\
\text { - Larger ratio between vertebral body and spinal canal (sagittal; all levels; } P<.0001 \text { ) } \\
\text { - Larger ratio between vertebral body and spinal canal (transverse; all levels; } P<.0007 \text { to } \\
\text { - } .0001 \text { ) } \\
\text { - Smaller sagittal SAC (except C3, C4; } P<.04 \text { to } P<.0001 \text { ) } \\
\text { - Smaller transverse SAC (except C6,C7; } \\
P<.03 \text { to } P=.002 \text { ) }\end{array}$ \\
\hline $\begin{array}{l}\text { Patel et al [8] } \\
(2012)\end{array}$ & Familial relationship & $\begin{array}{l}\text { Associations with CSM diagnosis (compared with controls): } \\
\text { - Excess of close relationships among CSM subjects }(P<.001) \\
\text { - First-degree relative with CSM (RR: } 5.21 ; 95 \% \mathrm{Cl}: 2.07-13.1) \\
\text { - Third-degree relative with CSM (RR: } 1.95 ; 95 \% \mathrm{Cl}: 1.04-3.7)\end{array}$ \\
\hline $\begin{array}{l}\text { Takamiya et al [3] } \\
\text { (2006) }\end{array}$ & $\begin{array}{l}\text { Age } \\
\text { Gender } \\
\text { Working years } \\
\text { Extension strain occupation }\end{array}$ & $\begin{array}{l}\text { Associations with CSM diagnosis (compared with those without myelopathy): } \\
\text { - Increased age (OR: 1.1; 95\% Cl: 1.01-1.14) }\end{array}$ \\
\hline $\begin{array}{l}\text { Wang et al [9] } \\
(2010)\end{array}$ & Vitamin D receptor gene polymorphisms & $\begin{array}{l}\text { Associations with CSM diagnosis (compared with controls): } \\
\text { - Apal genotype (OR: 2.88; 95\% Cl: 1.15-4.89) } \\
\text { - Taql genotype (OR: } 4.67 ; 95 \% \text { Cl: 2.33-5.76) }\end{array}$ \\
\hline $\begin{array}{l}\text { Yue et al [10] } \\
(2001)\end{array}$ & $\begin{array}{l}\text { Age } \\
\text { Gender Torg/Pavlov ratio }{ }^{\ddagger}\end{array}$ & $\begin{array}{l}\text { Associations with CSM diagnosis (compared with controls): } \\
\text { - Smaller Torg/Pavlov ratio }(P=.0001) \\
\text { - Increased age }(P=.002)\end{array}$ \\
\hline \multicolumn{3}{|c|}{ Poor quality studies (COE III), did not control for extraneous prognostic factors } \\
\hline $\begin{array}{l}\text { Chen et al [4] } \\
\text { (1994) }\end{array}$ & 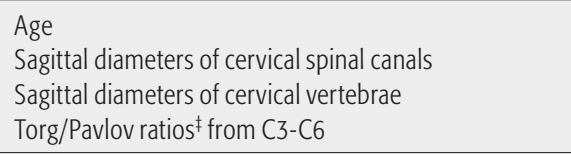 & $\begin{array}{l}\text { Associations with CSM diagnosis (compared with controls): } \\
\text { - Smaller sagittal diameter of cervical spinal canal }(P<.01) \\
\text { - Greater sagittal diameter of cervical vertebrae }(P<.005) \\
\text { - Smaller Torg/Pavlov ratio }(P<.001)\end{array}$ \\
\hline $\begin{array}{l}\text { Okada et al [7] } \\
(1994)\end{array}$ & $\begin{array}{l}\text { Transverse area of dural tube } \\
\text { Transverse area of spinal canal } \\
\text { Canal-occupying ratio of the spinal cord }\end{array}$ & $\begin{array}{l}\text { Associations with CSM diagnosis (compared with controls): } \\
\text { - Smaller spinal canal area at C3 }(P<.001) \\
\text { - Higher canal-occupying ratio of the spinal cord at C3 }(P<.001)^{\S}\end{array}$ \\
\hline
\end{tabular}

* CSM indicates cervical spondylotic myelopathy; CSA, cross-sectional area; CSF, cerebrospinal fluid; SAC, space available for spinal cord; RR, relative risk; $\mathrm{OR}$, odds ratio; and $\mathrm{Cl}$, confidence interval.

$\dagger<<.05$ and effect size estimates as reported by authors.

‡ Torg/Pavlov ratio was obtained by dividing the sagittal diameter of the cervical canal with the sagittal diameter of the cervical vertebra at the same level.

$\S$ Canal-occupying ratio of the spinal cord was not defined; unclear how it was measured. 
Table 3 Summary of sociodemographic factors and characteristics of the spinal cord, canal and vertebral body evaluated as risk factors for CSM reported in two or more studies.*

\begin{tabular}{|c|c|c|c|c|c|c|c|}
\hline & \multirow[b]{2}{*}{ Summary } & \multicolumn{4}{|c|}{$\begin{array}{l}\text { LoE III, controlled for extraneous prognostic } \\
\text { variables }\end{array}$} & \multicolumn{2}{|c|}{$\begin{array}{l}\text { LoE III, did not control } \\
\text { for extraneous } \\
\text { prognostic variables }\end{array}$} \\
\hline & & $\begin{array}{l}\text { Golash }[5]^{\dagger} \\
N=30\end{array}$ & $\begin{array}{l}\text { Hukuda }[6]^{\dagger \ddagger} \\
\mathrm{N}=85\end{array}$ & $\begin{array}{l}\text { Takamiya }[3]^{\dagger} \\
\mathbf{N}=368\end{array}$ & $\begin{array}{l}\text { Yue }[10]^{\dagger} \\
N=116\end{array}$ & $\begin{array}{l}\text { Chen }[4]^{\S} \\
\mathbf{N}=\mathbf{2 0 0}\end{array}$ & $\begin{array}{l}\text { Okada }[7]^{\S} \\
N=170\end{array}$ \\
\hline \multicolumn{8}{|l|}{ Sociodemographic } \\
\hline Increased age & Inconclusive & & & $\uparrow$ & $\uparrow$ & NS & \\
\hline Female gender & NS & & & NS & NS & & \\
\hline \multicolumn{8}{|c|}{ Cord, canal, vertebral body characteristics } \\
\hline Smaller spinal canal CSA & Inconclusive & NS & $\uparrow$ & & & & \\
\hline Larger vertebral body- sagittal diameter & $\uparrow$ & & $\uparrow$ & & & $\uparrow$ & \\
\hline Smaller spinal canal- transverse diameter & $\uparrow$ & & $\uparrow$ & & & & $\uparrow$ \\
\hline Smaller spinal canal- sagittal diameter & $\uparrow$ & & $\uparrow$ & & & $\uparrow$ & \\
\hline Smaller Torg/Pavlov ratio & $\uparrow$ & & & & $\uparrow$ & $\uparrow$ & \\
\hline
\end{tabular}

* CSM indicates cervical spondylotic myelopathy; NS, not significant; upward arrow, increased risk for diagnosis of CSM; and CSA, cross-sectional area.

+ Controlled for extraneous prognostic factors in multivariate regression analysis.

‡ Assessed gender, body height, body weight, and age on each variable, although no statistics were presented to verify controlling for prognostic factors and specifics of statistical modeling were not provided.

$\S$ Did not control for extraneous prognostic factors.

Table 4 Summary of factors evaluated as risk factors for CSM in isolated studies.*

\begin{tabular}{|c|c|c|c|c|c|c|}
\hline & \multicolumn{5}{|c|}{ LoE III, controlled for extraneous prognostic variables } & \multirow{2}{*}{$\begin{array}{l}\text { LoE III, did not control for } \\
\text { extraneous prognostic } \\
\text { variables } \\
\text { Okada }[7]^{\S} \\
\mathrm{N}=170\end{array}$} \\
\hline & $\begin{array}{l}\text { Golash }[5]^{\dagger} \\
N=30\end{array}$ & $\begin{array}{l}\text { Hukuda [6] } \\
\mathrm{N}=85\end{array}$ & $\begin{array}{l}\text { Patel }[8]^{\dagger} \\
N=1486\end{array}$ & $\begin{array}{l}\text { Takamiya } \\
{[3]^{\dagger} \mathrm{N}=368}\end{array}$ & $\begin{array}{l}\text { Wang }[9]^{\dagger} \\
\mathbf{N}=\mathbf{2 9 7}\end{array}$ & \\
\hline \multicolumn{7}{|l|}{ Sociodemographic } \\
\hline Greater working years & & & & NS & & \\
\hline Extension strain occupation & & & & NS & & \\
\hline \multicolumn{7}{|l|}{ Cord, canal, vertebral body characteristics } \\
\hline Smaller CSF space CSA & $\uparrow$ & & & & & \\
\hline Larger vertebral body-transverse diameter & & $\uparrow$ & & & & \\
\hline Larger CSA of vertebral body & & $\uparrow$ & & & & \\
\hline Larger vertebral body/spinal canal ratio (sagittal) & & $\uparrow$ & & & & \\
\hline Larger vertebral body/spinal canal ratio (transverse) & & $\uparrow$ & & & & \\
\hline Smaller sagittal SAC & & $\uparrow$ & & & & \\
\hline Smaller transverse SAC & & $\uparrow$ & & & & \\
\hline Cross-sectional SAC & & NS & & & & \\
\hline Dural tube transverse area & & & & & & NS \\
\hline Higher canal-occupying ratio of the spinal cord & & & & & & $\uparrow$ \\
\hline \multicolumn{7}{|l|}{ Inherited (genetic) factors } \\
\hline Relatives with CSM & & & $\uparrow$ & & & \\
\hline \multicolumn{5}{|l|}{ Vitamin D receptor gene polymorphism } & \multicolumn{2}{|l|}{$\uparrow$} \\
\hline \multicolumn{7}{|c|}{$\begin{array}{l}\text { CSM indicates cervical spondylotic myelopathy; CSA, cross-sectional area; CSF, cerebrospinal fluid; SAC, space available for spinal cord; NS, not } \\
\text { significant; and upward arrow, increased risk for diagnosis of CSM. } \\
\text { Controlled for extraneous prognostic factors in multivariate regression analysis. } \\
\text { Assessed gender, body height, body weight, and age on each variable, although no statistics were presented to verify controlling for prognostic factors } \\
\text { and specifics of statistical modeling were not provided. } \\
\text { Did not control for extraneous prognostic factors. }\end{array}$} \\
\hline
\end{tabular}




\section{CLINICAL GUIDELINES}

- Within the limits of our inclusion and exclusion criteria, no clinical guidelines were found that specifically address prognostic factors for CSM.

\section{EVIDENCE SUMMARY}

Table 5 What risk factors are associated with the presence (diagnosis) of cervical spondylotic myelopathy (CSM)?

\begin{tabular}{|c|c|c|c|c|c|}
\hline Prognostic factors & Strength & ence & & & Conclusions/comments \\
\hline 1. Age & Very low & Low & Moderate & High & $\begin{array}{l}\text { Increased age was associated with CSM in } 2 \text { studies and } \\
\text { found to be not associated with CSM diagnosis in } 1 \text { study }\end{array}$ \\
\hline 2. Female gender & Very low & Low & Moderate & High & Female gender was not associated with CSM in 2 studies \\
\hline 3. Spinal canal CSA & Very low & Low & Moderate & High & $\begin{array}{l}\text { Smaller cross-sectional area of the spinal canal was } \\
\text { associated with CSM in } 1 \text { study and was not associated with } \\
\text { CSM in } 1 \text { study }\end{array}$ \\
\hline $\begin{array}{l}\text { 4. Vertebral body sagittal } \\
\text { diameter }\end{array}$ & Very low & Low & Moderate & High & $\begin{array}{l}\text { Larger vertebral body sagittal diameter was associated with } \\
\text { CSM in } 2 \text { studies }\end{array}$ \\
\hline $\begin{array}{l}\text { 5. Spinal canal transverse } \\
\text { diameter }\end{array}$ & Very low & Low & Moderate & High & $\begin{array}{l}\text { Smaller spinal canal transverse diameter was associated } \\
\text { with CSM in } 2 \text { studies }\end{array}$ \\
\hline 6. Spinal canal sagittal diameter & Very low & Low & Moderate & High & $\begin{array}{l}\text { Smaller spinal canal sagittal diameter was associated with } \\
\text { CSM in } 2 \text { studies }\end{array}$ \\
\hline 7. Torg/Pavlov ratio & Very low & Low & Moderate & High & $\begin{array}{l}\text { Smaller Torg/Pavlov ratio was associated with CSM in } \\
2 \text { studies }\end{array}$ \\
\hline
\end{tabular}

\section{DISCUSSION}

- The major finding from this review was that a congenitally narrow spinal canal is a fundamental risk factor for the development of CSM. Multiple studies showed that various measurements reflecting congenital stenosis, including a larger vertebral body (sagittal diameter), smaller spinal canal (transverse and sagittal diameters), and a smaller Torg/Pavlov (T/P) ratio are associated with an increased risk of CSM. In 2009 Pavlov defined a $\mathrm{T} / \mathrm{P}$ ratio, the ratio of the sagittal diameter of the spinal canal to the anteroposterior diameter of the vertebral body, of 0.82 as indicative of congenital stenosis.
- Interestingly, a few single studies reported specific genetic factors that may be linked with the presence of CSM. One study reported a relationship between various polymorphisms of the vitamin $\mathrm{D}$ receptor gene and CSM, specifically patients who are ApaI "A" and Taq " $\mathrm{T}$ " allele carriers have an increased risk [9]. A genetic linkage study found an increased risk of CSM between both near and distant relatives [8].

- The independent influence of age on the development of CSM should be addressed in future studies. Two included studies suggested age was related to CSM, while a third study did not find an association. 
- The overall strength of evidence for various potential risk factors is very low (Table 5). Conclusions from this systematic review are limited by the lack of high-quality studies evaluating factors for CSM. The presence of CSM was based on varying diagnostic criteria provided by the authors of included articles. Additional limitations include disparate CSM case definitions across studies, which did not provide adequate control for potential confounders, limited assessment of true potential risk factors associated with disease, and study designs that prevented the ability to assess the temporality of potential risk factors. Documentation of subject selection and follow-up was poor in most studies.

- There is minimal evidence to suggest specific significant risk factors for CSM, and future research is warranted. In particular, it is important to determine factors that may predispose people to CSM to aid with directing appropriate preventive and management programs. Future research using populations with similar disease/ case definitions and methodologically rigorous study designs should be used to evaluate potential risk factors for the development of CSM.

\section{EDITORIAL PERSPECTIVE}

This is a high-quality systematic review that truly highlights the weakness of available evidence in defining risk factors for cervical spondylotic myelopathy. I recommend publication in its present form.

It is interesting to note that a smaller Torg/Pavlov ratio was found to be associated with CSM diagnosis, but not CSM development. It seems intuitive that patients with small Torg/Pavlov ratios at some point in their lives were not myelopathic, and at some point became myelopathic or developed myelopathy. Thus, it would seem that risk factors associated with CSM diagnosis would likely also be risk factors linked to CSM development. In any event, this is most likely reflective of low level of evidence available in the literature.

This systematic review would provide the basis for the background and introduction for a prospective longitudinal study examining risk factors for the development of CSM, or a prospective natural history study of patients with asymptomatic stenosis.

\section{REFERENCES}

1. Singh A, Choi D, Crockard A (2009) Use of walking data in assessing operative results for cervical spondylotic myelopathy: long-term follow-up and comparison with controls. Spine (Phila Pa 1976); 34(12):1296-1300.

2. Singh A, Crockard HA (1999) Quantitative assessment of cervical spondylotic myelopathy by a simple walking test. Lancet; 354(9176):370-373.

3. Takamiya Y, Nagata K, Fukuda K, et al (2006) Cervical spine disorders in farm workers requiring neck extension actions. J Orthop Sci; 11(3):235-240.

4. Chen IH, Liao KK, Shen WY (1994) Measurement of cervical canal sagittal diameter in Chinese males with cervical spondylotic myelopathy. Zhonghua Yi Xue Za Zhi (Taipei); 54(2):105-110.

5 Golash A, Birchall D, Laitt RD, et al (2001) Significance of CSF area measurements in cervical spondylitic myelopathy. Br J Neurosurg; 15(1):17-21.

6. Hukuda S, Xiang LF, Imai S, et al (1996) Large vertebral body, in addition to narrow spinal canal, are risk factors for cervical myelopathy. J Spinal Disord; 9(3):177-186.

7. Okada Y, Ikata T, Katoh S, et al (1994) Morphologic analysis of the cervical spinal cord, dural tube, and spinal canal by magnetic resonance imaging in normal adults and patients with cervical spondylotic myelopathy. Spine (Phila Pa 1976); 19(20):2331-2335.

8. Patel AA, Spiker WR, Daubs M, et al (2012) Evidence of an inherited predisposition for cervical spondylotic myelopathy. Spine (Phila Pa 1976); 37(1):26-29.

9. Wang ZC, Chen XS, Wang da W, et al (2010) The genetic association of vitamin $D$ receptor polymorphisms and cervical spondylotic myelopathy in Chinese subjects. Clin Chim Acta; 411(11-12):794-797.

10. Yue WM, Tan SB, Tan MH, et al (2001) The Torg-Pavlov ratio in cervical spondylotic myelopathy: a comparative study between patients with cervical spondylotic myelopathy and a nonspondylotic, nonmyelopathic population. Spine (Phila Pa 1976); 26(16):17601764. 